\title{
Temporal Predictability Facilitates Action, Not Perception
}

Psychological Science 24(7) 1335-1340

(C) The Author(s) 2013

Reprints and permissions:

sagepub.com/journalsPermissions.nav DOI: $10.1177 / 0956797612469411$ pss.sagepub.com

\section{Roland Thomaschke and Gesine Dreisbach}

Institute of Experimental Psychology, University of Regensburg

\begin{abstract}
Expectancy for upcoming action requirements is a fundamental prerequisite for human control of action. In the research reported here, we investigated which part of cognitive processing benefits from temporal predictability. In a binary forced-choice paradigm, visual targets were preceded by different intervals. In one condition, targets could be predicted by the length of the intervals. In other conditions, response goals or response effectors could be predicted by the length of the intervals. Behavioral advantages were observed when response effectors were temporally predictable, whereas temporal predictability of response goals and target stimuli was not sufficient. The findings thus show that temporal expectancy in speeded choice-reaction tasks facilitates late, effector-specific motor processing. These findings are of importance not only for our basic understanding of action control but also for any human-machine interaction that involves system delays.
\end{abstract}

\section{Keywords}

time estimation, time perception, motor processes, attention, temporal frequency

Received 12/16/11; Revision accepted 11/5/12

Expectancy for upcoming action requirements is a fundamental prerequisite for human control of action (Gilbert \& Wilson, 2007). This expectancy is almost always temporally specific (Thomaschke, Wagener, Kiesel, \& Hoffmann, 2011b). Different events are expected at different points in time. For example, when pressing a coffee vending machine's "Americano" button, I expect, because of my experience with the machine, that I will need to put my cup under the coffee nozzle after an interval of 2 to $3 \mathrm{~s}$. But if the interval after pressing the button appears to last approximately $10 \mathrm{~s}$ instead, I expect another event: I now expect an error message asking me to insert cash.

The temporal specificity of these expectancies is usually evidenced by suboptimal performance when expected events appear at atypical moments. Performance would be impaired if, because of a malfunction of the machine, the coffee nozzle spouted after the time the machine typically took to display an error message (i.e., $10 \mathrm{~s}$ ) or if the error message appeared at the time when the coffee was expected to be dispensed (i.e., 2-3 s; see Thomaschke, Wagener, Kiesel, \& Hoffmann, 2011a; Wagener \& Hoffmann, 2010). The expectancy described in this example has been termed specific temporal expectancy (Thomaschke, Kiesel, \& Hoffmann, 2011). Specific temporal expectancy can be defined as expectancy for a combination of an event and a point in time. An event is not expected per se, but is expected conditional on a point in time.

Specific temporal expectancy is prevalent in all areas of human behavior in which the duration of intervals is predictive about an event. The duration of pauses in oral speech, for example, affects listeners' expectancy about the complexity of the expressions that follow (Watanabe, Hirose, Den, \& Minematsu, 2008). Likewise, delay lengths in human-computer interaction are often predictive about the computer's response, and users adapt to these regularities. For example, people's optimism that a Web page will load successfully decreases continuously after they navigate to the URL until it eventually changes to the expectation of an error message (Seow, 2008).

\section{Corresponding Author:}

Roland Thomaschke, Institute of Experimental Psychology, University of Regensburg, Universitätsstraße 31, 93053 Regensburg, Germany E-mail: roland.thomaschke@psychologie.uni-regensburg.de 
Despite its prevalence in everyday life and its importance for goal-directed adaptive behavior, little is known about the underlying cognitive mechanisms of eventspecific temporal expectancy. Previous research on temporal cognition has focused mainly on general temporal expectancy, that is, adaptation to intervals that are not informative about specific events (Jepma, Wagenmakers, \& Nieuwenhuis, 2012; Lewis \& Miall, 2009; Los \& Schut, 2008).

An important question, pivotal to the further understanding of specific temporal expectancy, regards the cognitive locus of its influence: Which aspects of cognitive processing actually benefit from temporal predictability of events? Is it a certain stimulus that is expected after a certain interval, in the sense that the perceptual system processes stimuli more efficiently when they occur after their characteristic intervals? Or is it preparation of the response that benefits from specific temporal predictability? The latter option can be subdivided further. Most theories of motor control differentiate between the processing of distal action goals (e.g., choosing which nozzle to place the cup under) and the processing of motor programs to achieve these goals (e.g., moving the hand holding the cup; see Thomaschke, Hopkins, \& Miall, 2012). Both aspects of response processing might benefit from specific temporal expectancy.

To decide between these three alternative hypotheses (that specific temporal expectancy facilitates perception, that it facilitates action-goal processing, and that it facilitates movement processing), we devised a binary forcedchoice paradigm involving two target stimuli, four response buttons, and two modes of operating these buttons. The response buttons were grouped into two sets, one on the left and the other on the right, each consisting of an upper and a lower button. For one target, it was imperative to press an upper button; for the other target, participants had to press a lower button (see Fig. 1). Participants were instructed to alternate between the left set and the right set from trial to trial throughout the experiment. One group of participants operated button sets by switching hands and having four fingers (two fingers on each hand) resting on the buttons throughout the experiment (the bimanual group, see Fig. 2a). Another group operated both button sets with the same hand, moving it back and forth between the sets from trial to trial (the unimanual group; see Fig. 2b).

Each target was preceded by one of two possible intervals. Targets were temporally predictable: Overall, one of the targets appeared after one of the intervals with a probability of .7 , whereas the other target appeared after the other interval with the same probability. Critically, this correlation between target and interval was realized with only one of the response sets, the correlated set. When participants used this set, targets were preceded by their characteristic interval with a probability of .9 , whereas they were preceded unpredictably by both intervals with a probability of .5 for the uncorrelated set (see Fig. 1).

This arrangement allowed us to test whether specific temporal expectancy was specific to targets (perceptual expectancy), to response keys (action-goal expectancy), or to fingers (motor expectancy). On the basis of previous studies on temporal expectancy (Sanders, 1966; Zahn \& Rosenthal, 1966), we assumed that temporal predictability of an event could directly induce temporal expectancy for that event. This means that when a cognitive
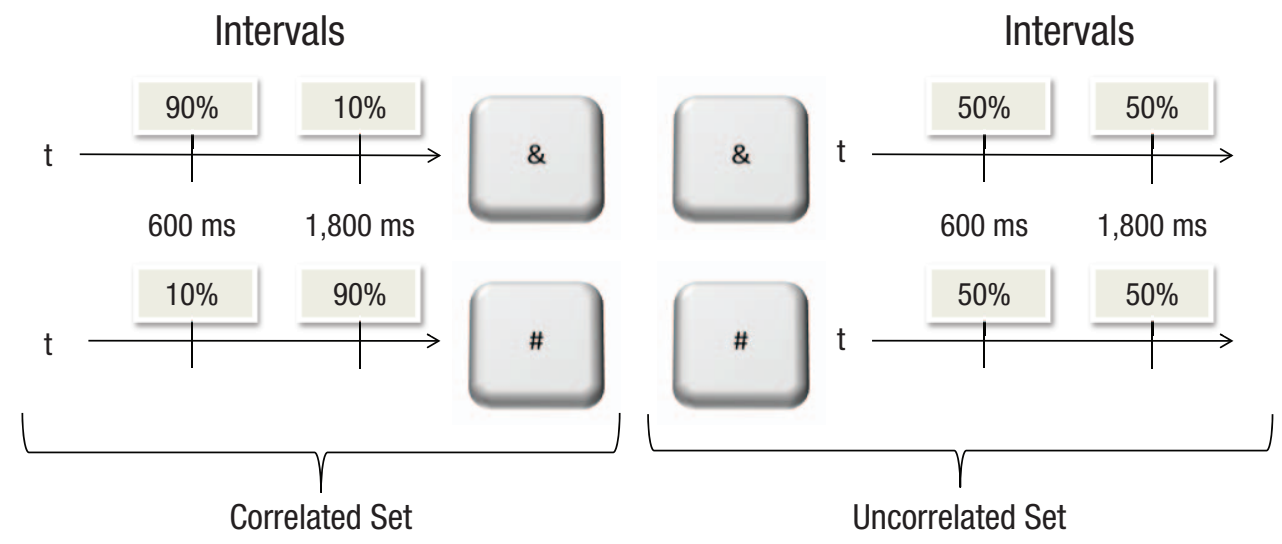

Fig. 1. Arrangement of button sets and frequencies of interval-button combinations. When the " $\&$ " symbol appeared, it was imperative to press the upper button of the current set; when the "\#" symbol appeared, it was imperative to press the lower button of the current set. Participants alternated between correlated and uncorrelated button sets from trial to trial. The four timelines illustrate the temporal probabilities separately for the four buttons. For the correlated button set, the interval predicted the identity of the upcoming symbol, and hence the required button, with $90 \%$ probability. For the uncorrelated button set, the interval was not predictive of the identity of the upcoming symbol. Note that in the experiment, the symbols appeared only on the screen (not on the buttons themselves). $\mathrm{t}=$ time. 
a

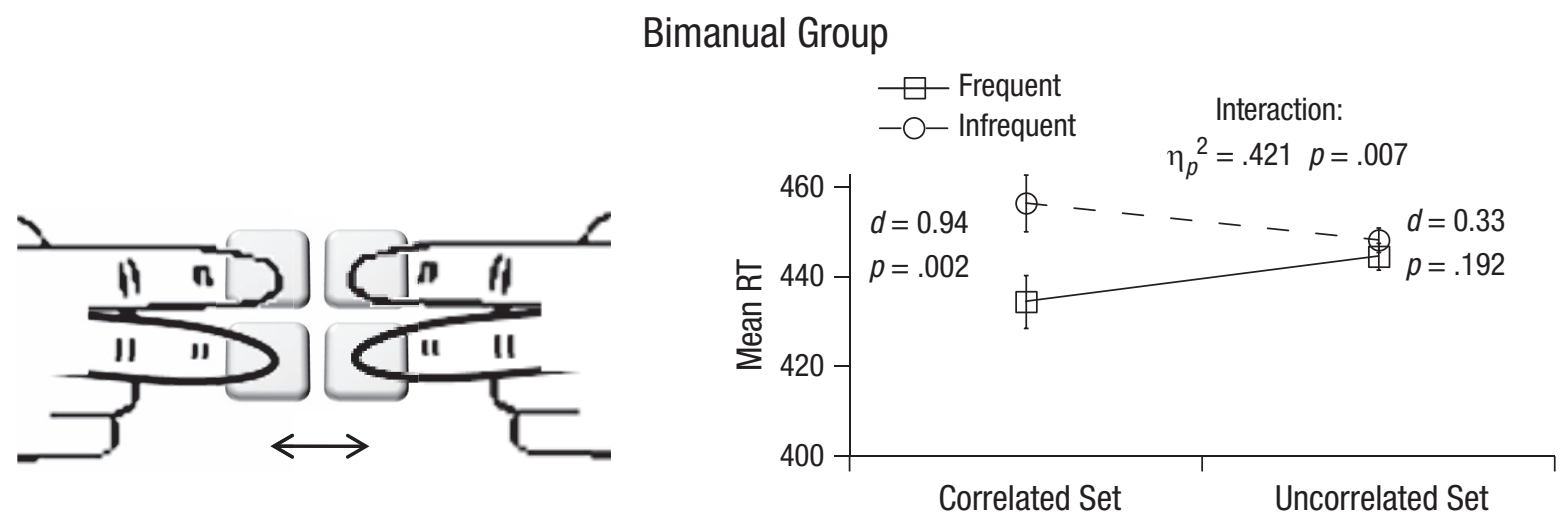

b

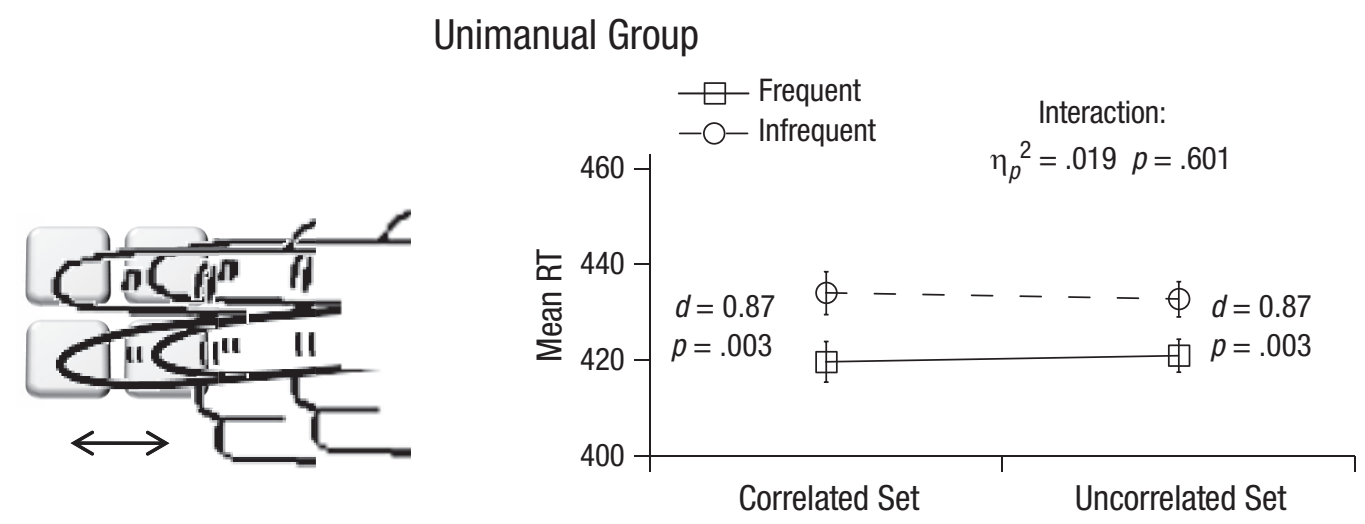

Fig. 2. Hand arrangements and results for the (a) bimanual and (b) unimanual groups. The illustrations show how participants in the two groups operated button sets with either two hands or one hand. The graphs show mean response times (RTs) as a function of button set and frequency of interval-target combination. Overlap of the confidence intervals represented by the error bars is equivalent to nonsignificance in a $t$ test with an alpha level of .05 (Tryon, 2001). Cohen's $d$ is standardized by difference scores (Gibbons, Hedeker, \& Davis, 1993) and refers to set-wise comparisons between frequent and infrequent interval-target combinations.

event (e.g., processing a stimulus or pressing a button with one's finger) occurs frequently after a certain interval, specific temporal expectancy should lead to shorter responses for that event after its typical, relative to its atypical, interval.

If temporal predictability facilitates target perception, a temporal-expectancy effect (i.e., faster responses for frequent combinations of interval and target than for infrequent combinations) should be observable irrespective of response set and response fingers. If, on the contrary, specific temporal expectancy facilitates the processing of action goals (i.e., of the selection of a particular response key), this effect should be restricted to the correlated button set (irrespective of the fingers used to operate the sets) because only response keys from that set are temporally predictable. If temporal expectancy is specific to individual fingers, then a temporal-expectancy effect would be restricted to those fingers that operate the correlated button set-that is, expectancy effects in this case should emerge in the unimanual group irrespective of the button set but should emerge in the bimanual group only for the fingers used to operate the correlated button set. Note that our hypotheses did not rely on participants' consciously detecting any interval-event regularities, because previous studies on the phenomenon have suggested that one adapts to interval-event frequencies in an implicit, nonconscious way (Thomaschke et al., 2011a, 2011b; Wagener \& Hoffmann, 2010).

\section{Method}

Participants (26 female, 5 male; mean age $=22.94$ years, $S D=2.82$ ) were allocated randomly to two experimental groups. All participants performed a speeded binarychoice task, responding with four response buttons to two target symbols (the "\&" symbol and the "\#" symbol), 
which appeared in random order. The four buttons were arranged in a square to form two response sets, one on the left and one on the right. Participants were instructed to switch between button sets after each trial throughout the experiment (see Fig. 2). One of the symbols was mapped to the upper buttons, and the other symbol was mapped to the lower buttons. This mapping was counterbalanced between participants. Participants operated the upper and lower buttons using their middle and index fingers, respectively. One group operated both sets with two hands so that their fingers remained on the same buttons throughout the experiment (bimanual group, $n=$ 15). The other group operated both button sets with their dominant hand so that the response hand moved from one set to the other between trials (unimanual group, $n=16)^{1}$

Target stimuli were preceded by one of two intervals (600 ms or 1,800 ms) during which a fixation cross was presented. When the correlated button set was used, one of the targets was preceded by the short interval in $90 \%$ of its occurrences, and the other target was preceded by the long interval in $90 \%$ of its occurrences. When the uncorrelated button set was used, both targets appeared equally often after both intervals. This procedure had the effect that each target was preceded by its typical interval in $70 \%$ of its occurrences. The experiment consisted of four blocks of 160 trials each. The experiment was preceded by a training phase, also consisting of four blocks, on the day before the main experiment. ${ }^{2}$ After the experiment, participants were asked whether they detected any regularity during the experiment.

\section{Results}

A mixed-factor analysis of variance (ANOVA) was conducted with the between-subjects factor group (bimanual vs. unimanual), the within-subjects factors button set (correlated vs. uncorrelated) and frequency of intervaltarget combinations (frequent vs. infrequent), and the dependent variable response time. The main effect for frequency of interval-target combinations was significant, $F(1,29)=35.143, p<.05$, as was the interaction between button set and frequency of combination, $F(1,29)=$ $7.752, p<.05$. Most important, the three-way interaction also was significant, $F(1,29)=4.355, p<.05$. To further qualify this interaction, two separate repeated measures ANOVAs were then conducted for both groups. The main effect for frequency was significant in the bimanual group, $F(1,14)=14.17, p=.002$, and in the unimanual group, $F(1,14)=22.17, p<.001$. More important, button set and frequency of interval-target combination interacted in the bimanual group, $F(1,14)=10.184, p<.05$, but not in the unimanual group, $F(1,15)=0.286$, n.s. (see Fig. 2). In an analogous ANOVA for error rates, no main effect or interaction was significant (all $p s>.110$ ). Note that participants responded faster overall in the unimanual group than in the bimanual group, probably because of the additional time needed to switch between hands in the bimanual condition (Rabbitt, 1978). According to Rabbitt (1978), this additional time might be related to the involvement of the nondominant hand in the bimanual condition. In a postexperimental interview, none of the participants reported being aware of the correlation between intervals and targets.

\section{Discussion}

Our results show that specific temporal expectancy for stimulus-response events is tied to individual effectors (in our experiment, fingers). When participants operated temporally predictable and temporally unpredictable keys with different fingers, temporal-expectancy effects were restricted to the fingers used to operate predictable keys. When participants operated those sets of keys with the same fingers, temporal expectancy effects were observed for both the temporally predictable and the temporally unpredictable keys. This effector specificity of temporal expectancy clearly excludes an explanation in terms of perceptual expectancy, because such an account would predict temporal-expectancy effects for any frequent interval-target combination, independent of response keys and response fingers. Thus, we conclude that specific temporal expectancy affects postperceptual processing stages.

The results also speak against temporal-predictability effects on action-goal processing. The temporal predictability of action goals was identical in both groups (i.e., only the correlated button set was predictable), but specific temporal-expectancy effects were clearly different between groups. Thus, the difference in expectancy must have been related to the processing of action features other than action goals.

One might speculate, however, that because of the different movement demands in both groups, participants' awareness of the interval-signal regularities differed, which would explain the performance difference between groups. The requirement to switch between hands in the bimanual group might have made participants more aware of the difference between button sets. Consequently, they might have altered their mode of adaptation into a more precise, fine-grained state, which in turn allowed button-set-specific adaptation to interval-event regularities in that group. However, for several reasons, we regard such an explanation as less plausible than our interpretation. First, we do not assume that temporal adaptation, a basic, ubiquitous, and evolutionarily important mechanism, is tightly connected to explicit detection of temporal regularities. Second, participants in both 
groups (unimanual and bimanual) uniformly reported not having detected any interval-signal regularities. Third, our explanation relies on only one assumption: Temporal predictability of a finger movement leads to temporal expectancy for that finger movement (independent of awareness of regularity). Thus, our interpretation is more parsimonious than an alternative explanation based on temporal-pattern discrimination by hand switch.

Consequently, we favor an explanation in terms of temporal expectancy of finger movements. With regard to the coffee-machine example from the introduction, our explanation means that it is the particular hand movement (of the cup-holding hand) that is temporally scheduled to the machine's typical response interval, not the choice of the appropriate nozzle to place the cup under and not the visual detection of the coffee spouting.

These conclusions are in line with similar results from the literature on nontemporal event expectancy. Precuing a stimulus-response event has been shown to facilitate mainly motor preparation (Leuthold \& Jentzsch, 2011; Leuthold, Sommer, \& Ulrich, 1996; Rosenbaum, 1980). In our study, the length of the interval might be seen as a temporal instance of a precue, scheduling specific motor preparation to certain points in time. Note, however, that we do not mean to exclude any specific temporal expectancy for cognitive processes other than late, effectorspecific response processing. In other contexts, for instance, in which target stimuli are difficult to discriminate, specific temporal expectancy might also facilitate perception (for examples of general temporal expectancy for stimuli, see Bueti, Bahrami, Walsh, \& Rees, 2010; Ghose \& Maunsell, 2002; Kingstone, 1992; Lange, Rösler, \& Röder, 2003; Rolke, 2008; Seibold, Bausenhart, Rolke, \& Ulrich, 2011).

In summary, our findings show that specific temporal expectancy in speeded choice-reaction tasks facilitates late, effector-specific motor processing, a finding that is of importance not only for our basic understanding of action control but also for any human-machine interaction that involves system delays.

\section{Declaration of Conflicting Interests}

The authors declared that they had no conflicts of interest with respect to their authorship or the publication of this article.

\section{Notes}

1. To avoid carryover effects with regard to correlation learning between conditions, each group was tested in only one of the conditions (unimanual or bimanual).

2. A training session was held because pilot studies have shown that the combination of button-set alternation and speeded choice response is difficult to learn. Data from the practice session were analyzed for explorative reasons. As would be expected from the complicated setup of the experiment, these practice data revealed no consistent pattern with regard to adaptation to the temporal regularities. We assumed that, in particular, the switch of the button sets between trials, which had to be paced by the participants, needed to be practiced more extensively. Obviously, participants needed to have acquired the ability to perform the task fluently and properly before they could adapt to the temporal regularities that were of interest to us. That the practice session was on the day before the main experiment opens the possibility that consolidation processes were involved here (Debas et al., 2010; Lewis, Couch, $\&$ Walker, 2011). These processes, however, were not the focus of our study.

\section{References}

Bueti, D., Bahrami, B., Walsh, V., \& Rees, G. (2010). Encoding of temporal probabilities in the human brain. Journal of Neuroscience, 30, 4343-4352. doi:10.1523/jneurosci $.2254-09.2010$

Debas, K., Carrier, J., Orban, P., Barakat, M., Lungu, O., Vandewalle, G., . . . Doyon, J. (2010). Brain plasticity related to the consolidation of motor sequence learning and motor adaptation. Proceedings of the National Academy of Sciences, USA, 107, 17839-17844. doi:10.1073/pnas 1012176107

Ghose, G. M., \& Maunsell, J. H. R. (2002). Attentional modulation in visual cortex depends on task timing. Nature, 419, 616-620. doi:10.1038/nature01057

Gibbons, R. D., Hedeker, D. R., \& Davis, J. M. (1993). Estimation of effect size from a series of experiments involving paired comparisons. Journal of Educational Statistics, 18, 271279.

Gilbert, D. T., \& Wilson, T. D. (2007). Prospection: Experiencing the future. Science, 317, 1351-1354. doi:10.1126/science .1144161

Jepma, M., Wagenmakers, E.-J., \& Nieuwenhuis, S. (2012). Temporal expectation and information processing: A model-based analysis. Cognition, 122, 426-441.

Kingstone, A. (1992). Combining expectancies. The Quarterly Journal of Experimental Psychology, 44A, 69-104. doi: 10.1080/14640749208401284

Lange, K., Rösler, F., \& Röder, B. (2003). Early processing stages are modulated when auditory stimuli are presented at an attended moment in time: An event-related potential study. Psychophysiology, 40, 806-817. doi:10.1111/14698986.00081

Leuthold, H., \& Jentzsch, I. (2011). Are temporal response features prepared in fixed order? Inferences from movementbased potentials. Psychophysiology, 48, 633-644.

Leuthold, H., Sommer, W., \& Ulrich, R. (1996). Partial advance information and response preparation: Inferences from the lateralized readiness potential. Journal of Experimental Psychology: General, 125, 307-323.

Lewis, P. A., Couch, T. J., \& Walker, M. P. (2011). Keeping time in your sleep: Overnight consolidation of temporal rhythm. Neuropsychologia, 49, 115-123. doi:10.1016/j .neuropsychologia.2010.10.025 
Lewis, P. A., \& Miall, R. C. (2009). The precision of temporal judgment: Milliseconds, many minutes, and beyond. Philosophical Transactions of the Royal Society B: Biological Sciences, 364, 1897-1905. doi:10.1098/rstb.2009.0020

Los, S. A., \& Schut, M. L. J. (2008). The effective time course of preparation. Cognitive Psychology, 57, 20-55.

Rabbitt, P. (1978). Hand dominance, attention, and the choice between responses. The Quarterly Journal of Experimental Psychology, 30, 407-416.

Rolke, B. (2008). Temporal preparation facilitates perceptual identification of letters. Perception \& Psychophysics, 70, 1305-1313. doi:10.3758/pp.70.7.1305

Rosenbaum, D. A. (1980). Human movement initiation: Specification of arm, direction, and extent. Journal of Experimental Psychology: General, 109, 444-474.

Sanders, A. F. (1966). Expectancy: Application and measurement. Acta Psychologica, 25, 293-313.

Seibold, V. C., Bausenhart, K. M., Rolke, B., \& Ulrich, R. (2011). Does temporal preparation increase the rate of sensory information accumulation? Acta Psychologica, 137, 56-64. doi:10.1016/j.actpsy.2011.02.006

Seow, S. C. (2008). Designing and engineering time: The psychology of time perception in software. Upper Saddle River, NJ: Addison Wesley.

Thomaschke, R., Hopkins, B., \& Miall, R. C. (2012). The Planning and Control Model (PCM) of motorvisual priming: Reconciling motorvisual impairment and facilitation effects. Psychological Review, 119, 388-407.
Thomaschke, R., Kiesel, A., \& Hoffmann, J. (2011). Response specific temporal expectancy: Evidence from a variable foreperiod paradigm. Attention, Perception, \& Psychophysics, 73, 2309-2322. doi:10.3758/s13414-011-0179-6

Thomaschke, R., Wagener, A., Kiesel, A., \& Hoffmann, J. (2011a). The scope and precision of specific temporal expectancy: Evidence from a variable foreperiod paradigm. Attention, Perception, E Psychophysics, 73, 953-964. doi:10.3758/s13414-010-0079-1

Thomaschke, R., Wagener, A., Kiesel, A., \& Hoffmann, J. (2011b). The specificity of temporal expectancy: Evidence from a variable foreperiod paradigm. The Quarterly Journal of Experimental Psychology, 64, 2289-2300. doi:10.1080/ 17470218.2011 .616212

Tryon, W. W. (2001). Evaluating statistical difference, equivalence, and indeterminacy using inferential confidence intervals: An integrated alternative method of conducting null hypothesis statistical tests. Psychological Methods, 6, 371-386.

Wagener, A., \& Hoffmann, J. (2010). Temporal cueing of targetidentity and target-location. Experimental Psychology, 57, 436-445. doi:10.1027/1618-3169/a000054

Watanabe, M., Hirose, K., Den, Y., \& Minematsu, N. (2008). Filled pauses as cues to the complexity of upcoming phrases for native and non-native listeners. Speech Communication, 50, 81-94.

Zahn, T. P., \& Rosenthal, D. (1966). Simple reaction time as a function of relative frequency of the preparatory interval. Journal of Experimental Psychology, 72, 15-19. 